\title{
The Secret Lives of Postnatally Depressed Dads
}

\section{Lloyd Frank Philpott}

School of Nursing and Midwifery, UCC

\section{Introduction}

Close your eyes and take a moment to think about postnatal depression (PND). What comes into your mind? What images present themselves?

Historically, PND has typically been associated with women. It has also been perceived as a product of biological causes. Consequently, most of the research to date has focused on mothers. However, researchers now conceptualise that PND is also the product of psychosocial causes such as sleep disturbance, relationship conflict and financial strain. It can therefore be presumed that such factors would also impact on the mental health of fathers or male partners in the postnatal period. The study of Paternal Postnatal Depression (PPND) is still in its infancy. However, an understanding of the problem has advanced considerably over the last ten years.

\section{Men, Fatherhood and PPND}

The transition and adaptation to fatherhood is considered one of the most acute transformations experienced during a man's life. The experience is influenced by personal, infant and environmental factors. Aside from the many joys, the postnatal period is marked by significant change and the absence of routine. Fathers experience many of the same changes and stresses that mothers do. While some fathers anticipate that they may find it difficult to adapt to parenthood, many fathers are not fully aware of the impact a baby will have on their lives.

Unprecedented social change in the previous four decades has seen a great shift in the role of fathers. This has resulted in the expectation that men play an equal and direct role in caring for their children. However, a great number of fathers have not had a role model from whom to learn appropriate fathering skills, having grown up in a time when men were either uninvolved or minimally involved in child-rearing. This has resulted in some men feeling inadequate and ill equipped as they begin their journey into fatherhood. This can subsequently cause PPND.

During the postnatal period (the period beginning immediately after the birth of a child and extending for six weeks) both mothers and fathers are at increased risk of depression. 
PND is a non-psychotic depressive disorder that occurs after the birth of a child and has traditionally been considered a disorder of women. Maternal PND (MPND) is a well-known condition that has been extensively researched and described in literature. In contrast, PPND is not widely acknowledged and not well researched. In general the mental health of fathers in the postnatal period is often not considered and this has resulted in men being under screened, underdiagnosed and undertreated for PPND and other postnatal mental health problems.

\section{What we know about PPND}

Prevalence: Estimates of the prevalence of PPND within the first year after the birth of a child vary widely ranging from $1.2 \%$ to $27.5 \%$. It is suggested that the wide statistical variation of estimates is related to the fact that different measurement tools are employed across studies. In addition, those studies that use the same measurement tools use different cut-off scores. An analysis of 43 PPND studies reported an average prevalence of 10.4\%. Thus far, only one Irish study on PPND has been undertaken. 100 fathers from the south of Ireland were screened for PPND in 2014 with the researchers finding a prevalence of $12 \%$.

Risk factors for PPND: Maternal depression has been identified as the most important predictor for PPND. Depression in one partner is significantly correlated with depression in the other. Fathers are more likely to be depressed if their partner is experiencing MPND. Having a history of clinical psychiatric treatment is another risk factor that is strongly linked to the development of PPND. It is suggested that this may be due to predisposing genetic factors and/or enduring environmental factors, with the perinatal period acting as a stressful life event which triggers remission.

The lack of choice and preparation inherent in an unplanned pregnancy also increases the risk of PPND. Other factors associated with a higher prevalence of PPND include unemployment, an unstable source of income, lower income levels, living in rented accommodation, low-income housing and overcrowded housing. Many of these demographic and socio-economic variables are associated and may act as indices of social deprivation.

Alternatively, social support networks act as a buffer and reduce the risk of PPND. It has been reported that fathers with higher levels of social support networks have a lower prevalence of PPND. Figure 1 outlined below highlights the risk factors for PPND.

Manifestation of PPND: PND in women is characterised by sad mood, apathy, anxiety, sleep disturbance, reduced self-esteem and difficulty coping with day-to-day tasks and mood. PND in men manifests itself differently and includes such symptoms as hostility, conflict and anger. In addition, men tend to withdraw or engage in escape activities such as overwork, sports, sex, gambling and alcohol abuse. 
Impact of PPND: The impact of MPND on infants and children is well documented. Recently, research has been conducted concerning the impact of PPND on infant and child development and well-being. Findings indicate a higher risk for increased family stress, lack of bonding, increased incidences of physical punishment and later child psychopathology such as emotional issues, conduct disorder and hyperactivity. Furthermore, the risk for negative parenting outcomes increases when both parents are depressed. Fathers play an important role in buffering their children from the effects of MPND. However, this buffer is lost when the father also has PND. When both parents are depressed infants are less likely to be put to sleep on their backs which increases the risk of sudden infant death (SIDS) or to be breastfed. Fathers are also less likely to play and interact with their infant if they are depressed.

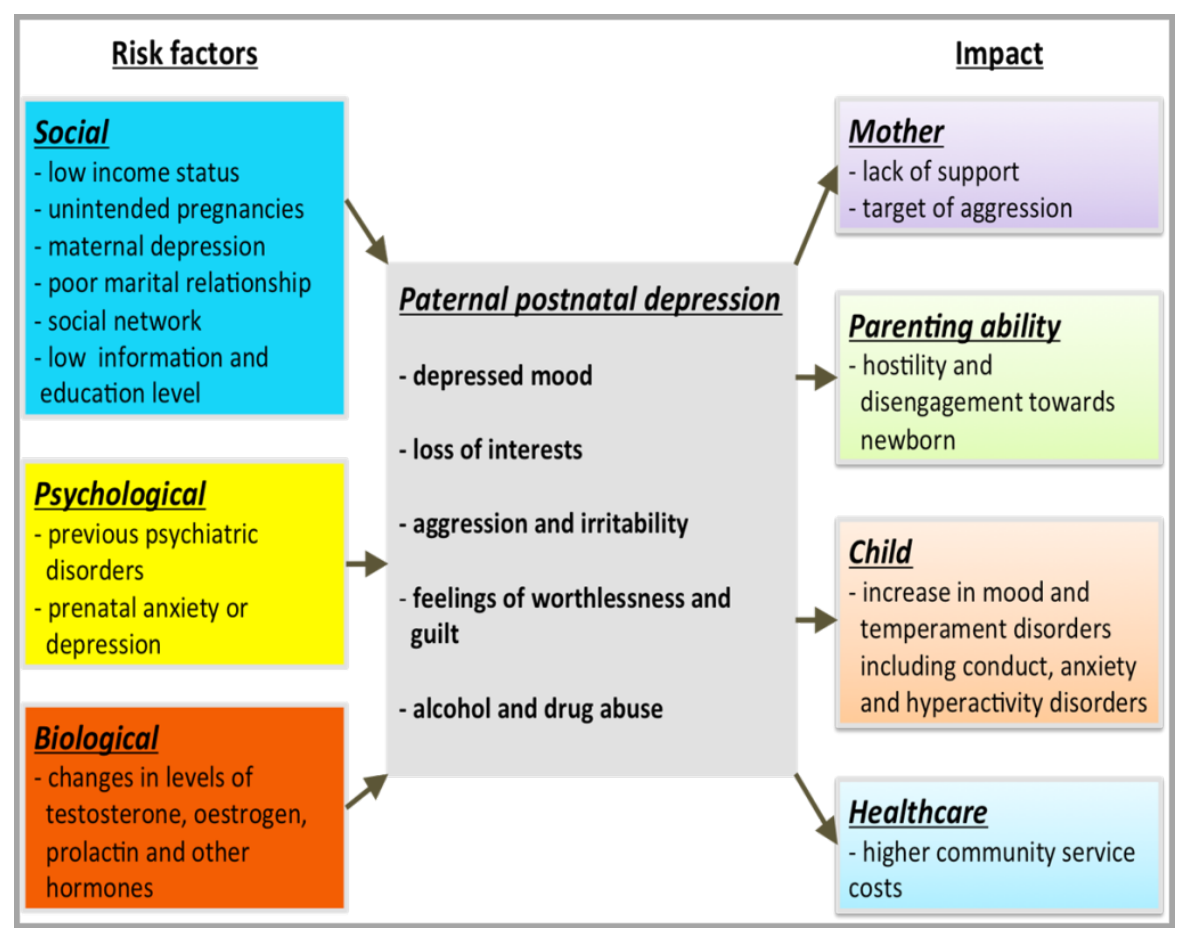

Figure 1: Risk factors, manifestations and impact of paternal postnatal depression. Source: Author

\section{What we don't know about PPND}

Research on PPND is still in its infancy so there are many aspects which have not yet been studied. Thus far the majority of PPND research has been undertaken in the US and Australia with white, middle class and married fathers. There is a paucity of knowledge in relation to PPND among fathers in non-traditional family settings i.e. stay at home fathers, non-biological fathers and single fathers and among fathers from minority ethnic groups and from different socioeconomic backgrounds. There is a need for research to be undertaken with fathers in these groups in order to identify their specific and unique risk factors. There is also a need for research to be carried out on the lived experiences of 
fathers with PPND.

\section{My Research Plan}

As part of my research, I plan to interview fathers who have been diagnosed with PPND. My research will give an insight into the lives of fathers who have experienced PPND. I will highlight each fathers experience before, during and after the birth of their infant, their self-recognition of symptoms of PPND, their help seeking behaviour, their treatment experiences and what they consider to be the ideal treatment for PPND. It is envisaged that the findings and subsequent reporting of the study will increase awareness of the problem and help fathers, their families and healthcare professionals better understand and recognise PPND.

\section{Conclusion}

PND has typically been associated with women. Consequently, most of the research to date has focused on mothers. However, research carried out over the last decade has revealed that PND can also impact on the mental health of fathers. Estimates of the prevalence of PPND within the first year postpartum are approximately 10\%. Several risk factors have been identified and these include living in rented accommodation, poor economic circumstances, not being married, having a history of depression and lack of social support. Despite an increase in research related to PPND over the last decade, the problem remains hidden and it is presently underscreened, underdiagnosed and undertreated.

Now close your eyes and take a moment to think about postnatal depression. What comes into your mind? What images present themselves?

I would like to thank my supervisors Dr. Patricia Leahy-Warren and Dr. Helen Mulcahy for their continued support. 